Check for updates

Cite this: RSC Adv., 2018, 8, 35448

\title{
Surface nanobubbles on the carbonate mineral dolomite $\uparrow$
}

\author{
Camilla L. Owens, (D) *a Edgar Schach, ${ }^{\mathrm{b}}$ Martin Rudolph ${ }^{\mathrm{b}}$ and Geoffrey R. Nash ${ }^{\mathrm{a}}$ \\ Surface nanobubbles are of wide interest to a number of research fields, ranging from mineral processing to \\ metamaterials. Their formation on hydrophobic surfaces has long been confirmed but the factors \\ controlling their size and location are less well understood. In this work we investigate, using non- \\ contact atomic force microscopy, the properties of surface nanobubbles on the mineral dolomite under \\ three aqueous solutions; water, depressant and collector. Nanobubbles were observed under all three \\ conditions, but with the highest density observed under collector conditions. Analysis of the critical \\ angle of the bubbles suggests that the collector does not affect the surface tension of the bubbles, but \\ instead does affect their pinning, consistent with the observed increased density.
}

Received 25th September 2018 Accepted 10th October 2018

DOI: 10.1039/c8ra07952h

rsc.li/rsc-advances deposits makes extraction difficult, and an improved understanding of the role of nanobubbles in the flotation process could lead to more efficient extraction.

\section{Experimental}

Non contact-atomic force microscopy (NC-AFM) was conducted on a Park Systems (South Korea) atomic force microscope XE100 located in Helmholtz Institute of Resource Technology Freiberg, Germany. The NC-AFM was combined with Raman Spectroscopy and an optical microscope to enable mineral identification and mapping. Images were produced in either $36 \mu \mathrm{m} \times 36 \mu \mathrm{m}$ or 8 $\mu \mathrm{m} \times 8 \mu \mathrm{m}$ sizes. Nanobubbles were generated using previously described air water supersaturation method. Both Contact cantilever (Park systems nanotechnology solutions partner) PPPCONTSCR 10M and ContAl-G Cantilever were used with a spring constant of $0.2 \mathrm{~N} \mathrm{~m}^{-1}$. ${ }^{4}$ The liquid cell used was of the same composition previous described by Rudolph and Peuker. ${ }^{10}$

The dolomite sample was analysed using X-ray diffraction and its composition confirmed with reference to the RTUFF database. ${ }^{18}$ High purity of the sample was calculated at $87.5 \%$. The dolomite sample was set in epoxy resin then machine polished. For NC-AFM measurements water with $\mathrm{KCl} 10^{-3} \mathrm{~mol} \mathrm{~L}^{-1}$ background electrolyte and a collector solution (of combined fatty acids (betacol) and hydroxamates (AM810) in usual proportions for flotation) were prepared before measurements and cooled to $5{ }^{\circ} \mathrm{C}$. This methodology was repeated with the water solution and depressant solution (combination of depressants from mineral processing). The exact composition of the depressant and collector is subject to non-disclosure. The liquid cell was filled by injecting up to $750 \mu \mathrm{L}$ of collector, water or depressant solution using a clean disposable plastic nozzle attached to a pipette. The same clean disposable plastic nozzles have previously been used by Babel and Rudolph, ${ }^{11}$ with plastic nozzles also being used in other studies. ${ }^{19,20}$ 
The liquid was then heated to between $20{ }^{\circ} \mathrm{C}$ and $30{ }^{\circ} \mathrm{C}$ for nanobubble measurements in a temperature controlled room of $21{ }^{\circ} \mathrm{C} \pm 1{ }^{\circ} \mathrm{C}$. Between measurements the cantilever was cleaned by washing in distilled water then ethanol and again distilled water. The dolomite surface was polished with DiaPro $\frac{1}{4} \mu \mathrm{m}$ diamond suspension by Struers to provide a fresh surface for each experiment. Subsequently, the surface was thoroughly washed with water, ethanol and water again to remove any residuals from the DiaPro $\frac{1}{4} \mu \mathrm{m}$ suspension or other sources of contamination. The dolomite sample was then further cleaned with sonication before being rinsed with deionized water as a last step.

\section{Results and discussion}

Nanobubbles were imaged on the surface of dolomite under water, collector and depressant conditions, as shown in Fig. 1. Image analysis from NC-AFM resulted in four good images, two images of nanobubbles in water, two images with nanobubbles in collector solution. To determine the density of the nanobubbles under different conditions, nanobubbles were identified from the images by comparing the phase and topography images from NC-AFM, which showed differences between nanobubbles and the mineral surface on the phase image. Nanobubbles were also identified by analysing their measured cross sections. Four cross sections of the nanobubbles were extracted and fitted to a spherical cap, ${ }^{\mathbf{1 4}}$ as shown in Fig. 2, following the methodology proposed by Rangharajan et al. ${ }^{21}$ and Li et al. ${ }^{22}$ Fitting was conducted using non-linear least squares, with the height and lateral length as fitting parameters (see ESI $\dagger$ for more details). In total,
31 nanobubbles were identified from 63 possible candidates (ESI $\dagger$ ) (nanobubbles had to show both phase difference and good fitting to cross sections to be identified as a nanobubble). The extracted nanobubble height ranged from, $73.9 \mathrm{~nm}$ to $7.8 \mathrm{~nm}$, which compares to the value of $1.4 \mathrm{~nm}$ obtained for the root mean square of the surface roughness. Bubble density was calculated by dividing the number of bubbles by surface area, and under collector conditions the nanobubble density was 0.656 per $\mu \mathrm{m}^{2}$. In contrast the nanobubbles in water conditions had a bubble density of 0.342 nanobubbles per $\mu \mathrm{m}^{2}$, with depressants much lower at 0.0625 nanobubbles per $\mu \mathrm{m}^{2}$.

Contact angles were extracted from the cross section fits, correcting for the size of the cantilever tip. In this study, a cantilever with a tip radius of $7 \mathrm{~nm}$ was used, ${ }^{23,24}$ and correction of the contact angle was calculated following methodology from Wang et $a .^{29}$ Nanobubbles in the collector reagent scheme had an average contact angle of $9.74^{\circ}$ with standard deviation \pm 3.07 , whereas those in water conditions had an average contact angle of $15.14^{\circ}$ with standard deviation of $\pm 9.27^{\circ}$, as plotted in box and whisker format in Fig. 3. The collector contact angle was the average of 21 nanobubble contact angles with the water being an average of 11 nanobubble values.

The amount of supersaturation $(\xi)$ of the liquid has also been proven to clearly effect both contact angle, $\theta$, and the lateral length of the nanobubble, with Lohse and Zhang ${ }^{14}$ showing that for a (a fixed) gas oversaturation $\zeta>0$, there exists a stable equilibrium defined by:

$$
\sin (\theta)=\xi \frac{P_{0} L}{4 \sigma}=\xi \frac{L}{L_{\mathrm{c}}}
$$

where $\theta$ is the contact angle, $L$ the lateral length, and $L_{\mathrm{c}}$ the critical lateral extension $\left(L_{\mathrm{c}}=4 \sigma / P_{0} \approx 2.84 \mu \mathrm{m}\right),{ }^{14}$ where $\sigma$ is the
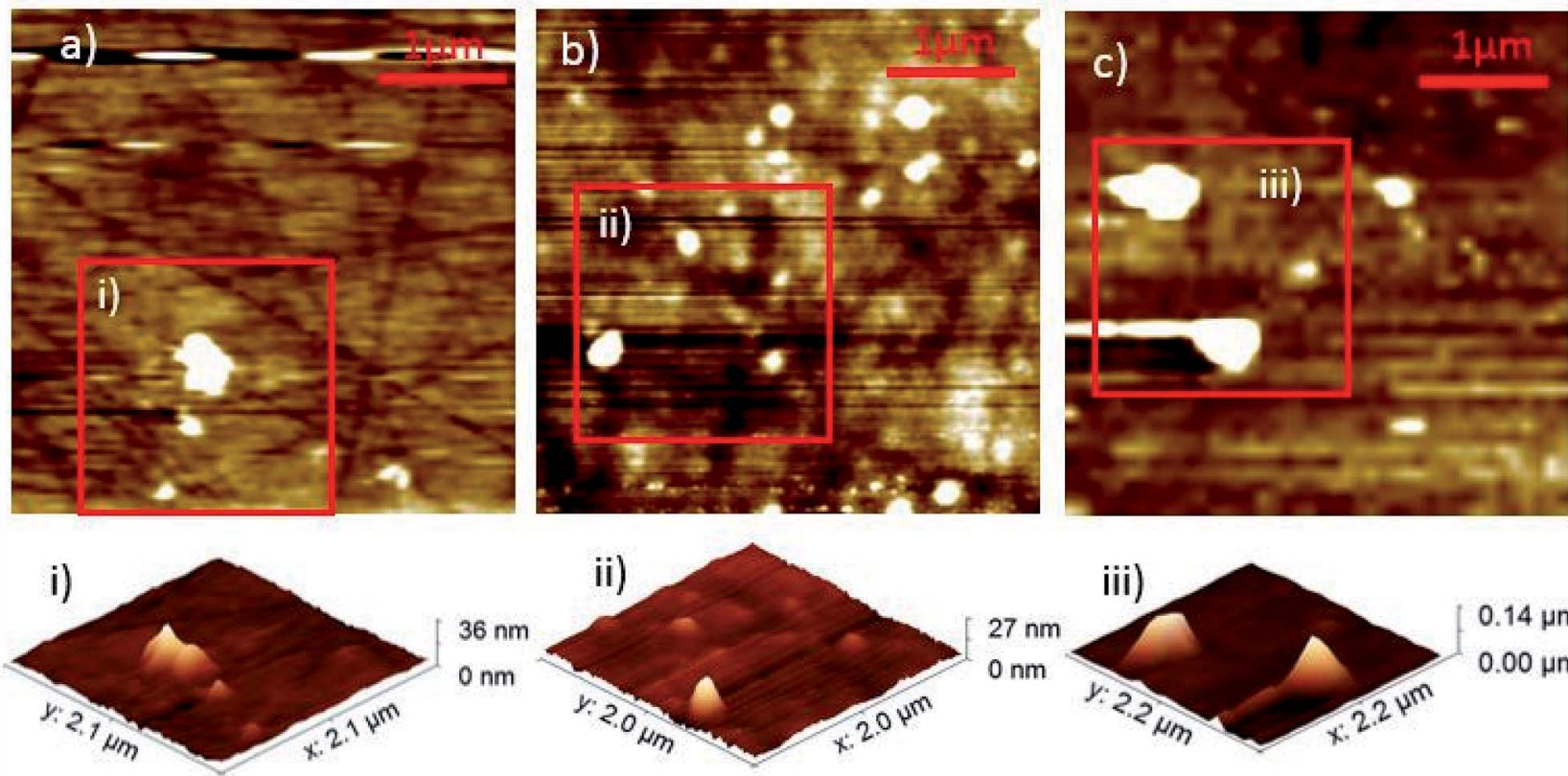

Nanobubbles in collectors

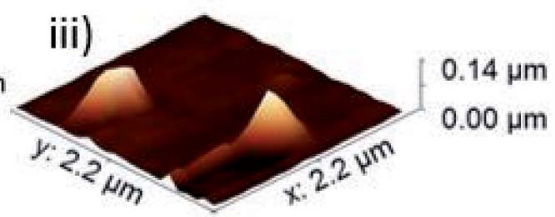

\section{Nanobubbles in water}

Fig. 1 Nanobubble density differences with topographical AFM images of nanobubbles in depressant (a) collectors (b) and water (c). Each image is $4 \mu \mathrm{m} \times 4 \mu \mathrm{m}$ across. 


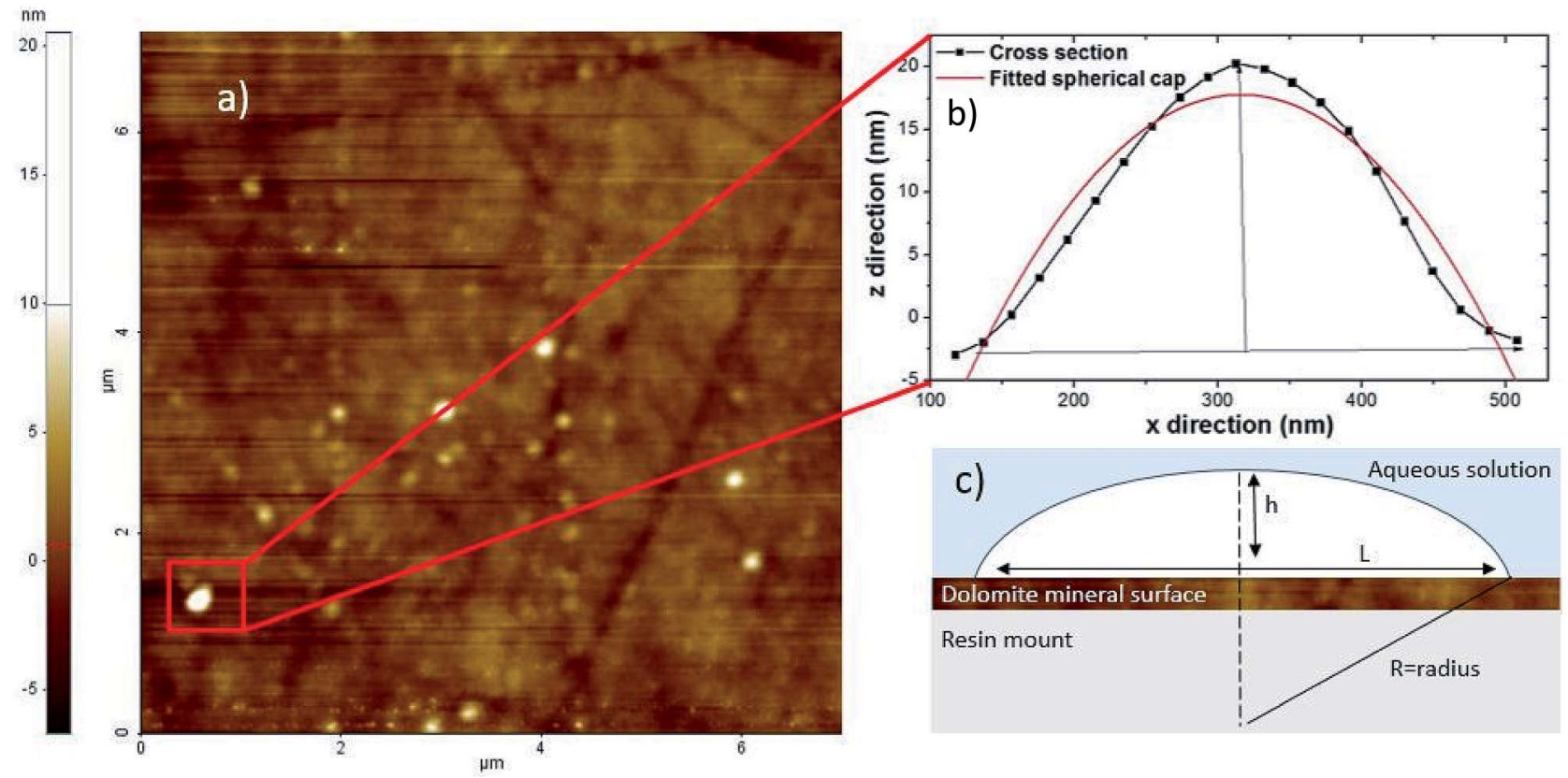

Fig. 2 (a) $8 \mu \mathrm{m} \times 8 \mu \mathrm{m}$ non contact atomic force microscopy (AFM) image of surface nanobubbles on the carbonate mineral dolomite (b) cross section of nanobubble height and width extracted from the non contact AFM image (c) diagram of height $(h)$, length $(L)$ and radius $(R)$ with contact angle $(\theta)$ of the nanobubble. Diagram (c) after Lohse and Zhang. ${ }^{14}$

surface tension and $P_{0}$ the ambient pressure). Although reagents such as fatty acids have been shown to effect surface tension in macroscopic bubbles, ${ }^{25,26}$ the contact angle and therefore surface tension of nanobubbles does not necessarily appear to be effected by reagents. ${ }^{27,28}$ In Fig. 4, the sine of the contact angle is plotted as a function of the nanobubble length under both water and collector conditions. In both cases $\sin (\theta)$ shows an approximately linear dependence on the length (the straight lines shown are linear fits to the data), consistent with eqn (1). Values of the ratio of $\xi / L_{\mathrm{c}}$ of $0.6 \mu \mathrm{m}^{-1}$ and $0.5 \mu \mathrm{m}^{-1}$, for nanobubbles in water

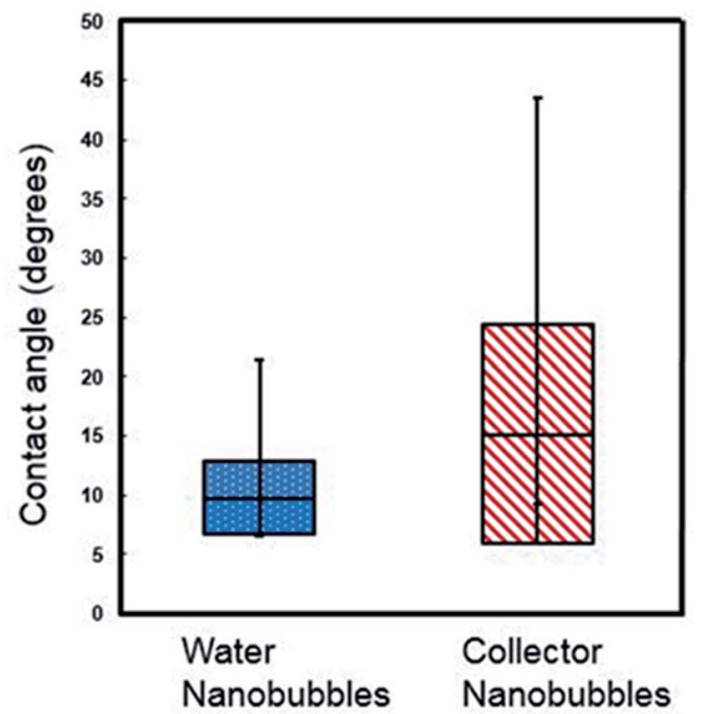

Fig. 3 Box and whisker plot of contact angles of nanobubbles under collector and DI water conditions (the data was selected using fitting of a spherical cap model after Lohse and Zhang. ${ }^{14}$ and collector respectively, were extracted from gradient (eqn (1)) of the linear fits shown. Wang et al. ${ }^{29}$ obtained a value of $\xi / L_{\mathrm{c}}$ of $2.9 \mu \mathrm{m}^{-1}$, for nanobubbles induced in nanopits, which coupled with a value of $L_{\mathrm{c}}$ of $2.84 \mu \mathrm{m}$, led to an estimated oversaturation $\xi$ of 8.2. In our case, taking the same value of $L_{\mathrm{c}}$, the gradient leads to an estimated oversaturation $\xi$ of 1.7 under water conditions. The value of oversaturation obtained in this work therefore seems reasonable given the different methodologies in which nanobubbles were induced in these studies.

In addition, there is no significant difference between the values of $\xi / L_{\mathrm{c}}$ obtained under water and collector conditions. Assuming that the over-saturation and the ambient pressure where the same in both cases, this also implies that the surface tension was not affected by the collector. This is consistent with the thin film model of nanobubbles developed by Zhang

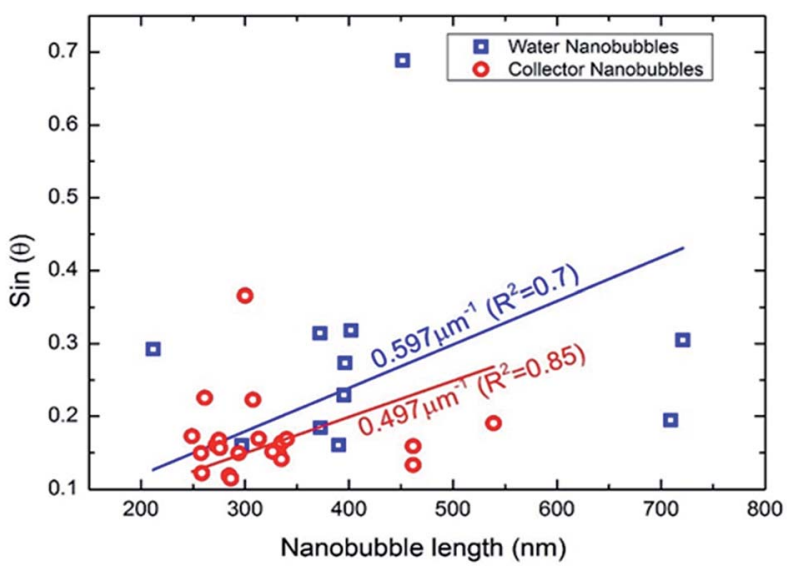

Fig. 4 Sine of the contact angle as a function of lateral length under water and collector conditions. 
et al. ${ }^{27,30}$ However, the difference in the density of bubbles observed under water and collector conditions suggests that the collector has affected the pinning of the bubbles, which in turn determines the lateral length. Previous work by Xiao et al. ${ }^{31}$ investigating the stability of nanobubbles under surfactants has shown with molecular dynamics simulations that the contact angle does not depend on pinning, whereas the density of nanobubbles does. ${ }^{31}$ This is consistent with earlier results by Mikhlin et al. ${ }^{8}$ who investigated nanobubbles at the surface of the sulphide mineral galena $(\mathrm{PbS})$ and found that the number of nanobubbles increased after the surface had been pre-treated using a xanthate collector rather than with water. Tan et al. ${ }^{32}$ investigated the exact value of the pinning force by using an AFM tip to deform nanobubbles whilst imaging the mechanical response using total internal reflection fluorescence microscopy (TIRFM). The pinning strength varied between $5 \mathrm{mN} \mathrm{m}^{-1}$ to 20 $\mathrm{mN} \mathrm{m}^{-1}$, with the variation attributed to chemical and physical heterogeneities of the surface. The relative smoothness of the mineral surface in this case indicates that it is chemical heterogeneities induced by the collector that affects the bubble pinning. Previous work by $\mathrm{Xie}^{33}$ has shown non uniform adsorption of xanthate on sphalerite caused differing regions of hydrophobicity on the mineral surface.

Finally, the increase in the observed number of nanobubbles under collector conditions is also consistent with macroscopic studies investigating micro flotation for minerals processing. Both Espiritu and Waters ${ }^{16}$ and Azizi and Larachi ${ }^{34}$ investigated micro flotation of dolomite under collector (hydroxamic acid and fatty acid). In Azizi and Larachi, ${ }^{34} 75.3 \%$ of dolomite was recovered (floated) with hydroxamic collector compared to $4.1 \%$ of dolomite was floated (recovered) under water conditions. Surface nanobubbles could therefore be playing an important part in froth flotation, although much work is required to quantify the significance of this contribution. Future work may focus on more minerals and reagent regimes, in particular rare earths were processing is of such importance to the viability of deposits. ${ }^{17}$

\section{Conclusions}

Surface nanobubbles on dolomite, induced using the air water supersaturation method, were imaged using NC-AFM under water, collector and depressant surfactant conditions. The observed bubble density was highest under collector conditions, with 0.656 bubbles per $\mu \mathrm{m}^{2}$, compared to 0.342 nanobubbles per $\mu \mathrm{m}^{2}$ under water conditions. Analysis of the bubble contact angles, which were extracted by fitting a spherical cap to the nanobubbles, suggests that the collector does not affect the surface tension, but does affect their pinning. This is consistent with both the observed bubble density, but also with macroscopic flotation studies. These results there lay the foundation for an improved understanding of the role of nanobubbles in the flotation process.

\section{Conflicts of interest}

There are no conflicts to declare.

\section{Acknowledgements}

This research was conducted thanks to funding by the UK's Natural Environment Research Council SoS RARE Grant agreement no. NE/M 011429/1 and Mkango Resources Ltd. Work could not have been conducted without collaboration between Helmholtz Institute Freiberg for Resource Technology (HIF), Germany and SoS Rare (University of Exeter). Special thanks to Tom Leistner, Nathalie Kupka, Hoang Huu Duong and Bent Matthias Babel for their assistance at HIF. Thanks to Robert Möckel and Doreen Ebert for XRD analysis. C L Owens is grateful for Lisa Ditscherlein of Technische Universität Bergakademie Freiberg for helpful and insightful discussions on the measurement of nanobubbles. Support was also provided by Frances Wall, Camborne School of Mines, University of Exeter.

\section{Notes and references}

1 S. T. Lou, Z. Q. Ouyang, Y. Zhang, X. J. Li, J. Hu, M. Q. Li and F. J. Yang, J. Vac. Sci. Technol., B: Microelectron. Nanometer Struct.-Process., Meas., Phenom., 2000, 18, 2573-2575.

2 N. Ishida, T. Inoue, M. Miyahara and K. Higashitani, Langmuir, 2000, 16, 6377-6380.

3 J. H. Weijs and D. Lohse, Phys. Rev. Lett., 2013, 110, 054501. 4 L. Ditscherlein, J. Fritzsche and U. A. Peuker, Colloids Surf., A, 2016, 497, 242-250.

5 L. Wang, X. Miao and G. Pan, Langmuir, 2016, 32, 1114711154.

6 H. An, B. H. Tan, J. G. S. Moo, S. Liu, M. Pumera and C. D. Ohl, Nano Lett., 2017, 17, 2833-2838.

7 M. A. Hampton, B. C. Donose and A. V. Nguyen, J. Colloid Interface Sci., 2008, 325, 267-274.

8 Y. L. Mikhlin, A. A. Karacharov and M. N. Likhatski, Int. J. Miner. Process., 2015, 144, 81-89.

9 M. Alheshibri, J. Qian, M. Jehannin and V. S. Craig, Langmuir, 2016, 32, 11086-11100.

10 M. Rudolph and U. A. Peuker, Miner. Eng., 2014, 66, 181-190. 11 B. Babel and M. Rudolph, Miner. Eng., 2018, 121, 212-219.

12 V. S. J. Craig, J. Colloid Interface Sci., 1996, 183, 260-268.

13 P. N. Bhandari, Y. Cui, B. D. Elzey, C. J. Goergen, C. M. Long and J. Irudayaraj, Sci. Rep., 2017, 7, 1-14.

14 D. Lohse and X. Zhang, Phys. Rev. E, 2015, 91, 031003.

15 S. Calgaroto, K. Q. Wilberg and J. Rubio, Miner. Eng., 2014, 60, 33-40.

16 E. R. L. Espiritu and K. E. Waters, Miner. Eng., 2018, 116, 101-106.

17 F. Wall, A. Rollat and R. S. Pell, Elements, 2017, 13, 313-318. 18 B. Lafuente, R. T. Downs, H. Yang and N. Stone, Highlights in Mineralogical Crystallography, De Gruyter, 2016.

19 P. Knüpfer, J. Fritzsche, T. Leistner, M. Rudolph and U. A. Peuker, Colloids Surf., A, 2017, 513, 215-222.

20 L. Ditscherlein, J. Fritzsche and U. A. Peuker, Colloids Surf., A, 2016, 497, 242-250.

21 K. K. Rangharajan, K. J. Kwak, A. T. Conlisk, Y. Wu and S. Prakash, Soft Matter, 2015, 11, 5214-5223.

22 D. Li, D. Jing, Y. Pan, W. Wang and X. Zhao, Langmuir, 2014, 30, 6079-6088. 
23 H. Teshima, K. Takahashi, Y. Takata and T. Nishiyama, J. Appl. Phys., 2018, 123, 054303.

24 W. Walczyk and H. Schönherr, Langmuir, 2014, 30, 1195511965.

25 I. M. Hauner, A. Deblais, J. K. Beattie, H. Kellay and D. Bonn, J. Phys. Chem. Lett., 2017, 8, 1599-1603.

26 A. Atrafi and M. Pawlik, Miner. Eng., 2016, 85, 138-147.

27 X. H. Zhang, N. Maeda and V. S. Craig, Langmuir, 2006, 22, 5025-5035.

28 W. A. Ducker, Langmuir, 2009, 25, 8907-8910.
29 Y. Wang, X. Li, S. Ren, H. T. Alem, L. Yang and D. Lohse, Soft Matter, 2017, 13, 5381-5388.

30 X. Zhang, M. H. Uddin, H. Yang, G. Toikka, W. Ducker and N. Maeda, Langmuir, 2012, 28, 10471-10477.

31 Q. Xiao, Y. Liu, Z. Guo, Z. Liu and X. Zhang, Appl. Phys. Lett., 2017, 111, 131601.

32 B. H. Tan, H. An and C. D. Ohl, Phys. Rev. Lett., 2017, 118, 054501.

33 L. Xie, 2017, Doctoral thesis, University of Alberta.

34 D. Azizi and F. Larachi, Colloids Surf., A, 2018, 537, 126-138. 